\title{
Anestesia convencional e técnica de tumescência em cadelas submetidas à mastectomia. Avaliação da dor pós-operatória
}

[Conventional anesthesia and tumescent technique in bitches which underwent mastectomy. Evaluation of postoperative pain]

\author{
C.S. Aguirre, B.W. Minto*, E.G. Faria, M. Horr, F.G.F. Filgueira, A.B. Nardi
}

Faculdade de Ciências Agrárias e Veterinárias - Universidade Estadual Paulista - Unesp - Jaboticabal, SP

\section{RESUMO}

As neoplasias mamárias são as mais comuns em cadelas e geralmente acometem fêmeas de meia-idade a idosas, não castradas ou que foram submetidas ao procedimento de ovario-histerectomia tardiamente. A principal forma de tratamento é a excisão cirúrgica, sendo a ressecção unilateral das glândulas mamárias o procedimento mais realizado na prática veterinária. $\mathrm{O}$ objetivo do presente estudo foi comparar a dor pós-operatória em cadelas submetidas ao procedimento de mastectomia unilateral sob efeito das técnicas anestésicas de tumescência ou convencional. Foram utilizadas 20 cadelas, divididas em dois grupos: um grupo recebeu apenas a anestesia geral inalatória (grupo convencional), e o outro recebeu adicionalmente a anestesia infiltrativa por tumescência (grupo tumescência). Elas foram avaliadas nas primeiras 24 horas do período pós-operatório, e o processo álgico foi avaliado pela escala de dor da Universidade de Melbourne. As médias dos escores obtidos ao longo do tempo foram submetidas ao teste de Tukey a um nível de 5\% de significância $(\mathrm{p}<0,05)$. Não foram observadas diferenças significativas entre os grupos com relação à dor pós-operatória. A técnica anestésica infiltrativa por tumescência apresentou a vantagem da redução do sangramento transoperatório e mostrou-se exequível em pequenos animais, entretanto seu uso está relacionado à experiência e à preferência do cirurgião e do anestesista.

Palavras-chave: cães, tumor mamário, cirurgia, dor, tumescência

\begin{abstract}
Mammary tumors are the most common neoplasm in bitches. Intact, mild to advanced aged female dogs are generally more affected. The main treatment is surgical excision and unilateral mastectomy is the most performed procedure in veterinary practice. The aim of this study was to compare the postoperative pain in dogs which underwent unilateral mastectomy and were anesthetized with tumescence and conventional anesthesia. Twenty bitches were randomly divided into two groups: Conventional Group $(G C)$, which received only general inhalational anesthesia and Tumescence Group (GT), which also received tumescence anesthesia. All dogs were evaluated during the first 24 hours postoperatively. The scale of Pain from the University of Melbourne was used for the evaluation. The tukey test at a $5 \%$ level of significance ( $p<0.05)$ was used. There were no significant differences between groups related to postoperative pain. The technique of tumescent anesthesia reduced bleeding during surgery and is feasible in dogs. Its use is related to the surgeon and anesthesiologist's experience and preferences.
\end{abstract}

Keywords: dogs, mammary tumor, surgery, pain, tumescence

\section{INTRODUÇÃO}

As neoplasias mamárias são as mais comuns nas cadelas (Morris e Dobson, 2001), constituindo
$52 \%$ de todos os tumores que as afetam (Queiroga e Lopes 2002). Vários estudos aduzem uma incidência de aproximadamente 200 casos em 100 mil cães a cada ano (Vascellari et al., 2009).

Recebido em 10 de junho de 2013

Aceito em 12 de dezembro de 2013

*Autor para correspondência (corresponding author)

E-mail: brunominto@fcav.unesp.br 
O risco relativo de desenvolver neoplasia mamária é maior em fêmeas não castradas ou que foram submetidas à castração tardiamente (Headlund, 2008; Johnson, 2010), em especial após os seis anos de idade (Davis e Stone, 2008). Apesar disso, a etiologia definitiva da doença não é completamente entendida, embora haja evidências de dependência hormonal, visto que a incidência é menor em cadelas que foram submetidas à ovario-histerectomia precoce (Otoni et al., 2010).

Os pares de mamas caudais são os mais acometidos, provavelmente por possuírem maior quantidade de tecido mamário (Van Nimwegen e Kirpensteijn, 2012). Ademais, histologicamente, cerca de $50 \%$ dos tumores mamários em cadelas são malignos (Queiroga e Lopes, 2002; Stratmann, 2008), sendo a sua maioria representada pelos carcinomas (Daleck et al., 1998).

O tratamento para a maioria dos tumores mamários é a excisão cirúrgica (Morris e Dobson, 2001), com exceção daqueles portadores de doença metastática grave (Van Nimwegen e Kirpensteijn, 2012), tumores inoperáveis ou carcinomas inflamatórios (Headlund, 2008). Inúmeras técnicas de mastectomia, da lumpectomia à mastectomia radical bilateral, são descritas, e a escolha pela melhor técnica depende do tamanho e da localização das massas, do estado do paciente, do tipo histológico e da preferência do cirurgião (Van Nimwegen e Kirpensteijn, 2012), visto que o tipo de cirurgia não influencia no tempo de sobrevida do animal e/ou no tempo de sobrevida livre da neoplasia. Entretanto, são recomendadas mastectomias amplas com margens efetivas de segurança (White, 2007).

A mastectomia radical, procedimento comumente indicado, é considerada invasiva e extensiva, que resulta em edema, inflamação e processo álgico de ocorrência moderada a intensa (Gakiya et al., 2011).

A dor pós-operatória tem efeitos mórbidos em pequenos animais e pode comprometer a recuperação (Sarrau et al., 2007). Uma analgesia adequada é essencial para garantir um rápido e bem-sucedido resultado pós-operatório (Sarrau et al., 2007). A dor traz malefícios, como o aumento dos níveis de cortisol, induzindo o organismo a uma resposta de estresse, o que pode resultar em hiperglicemia, taquicardia, hipertensão, arritmias ventriculares, que podem interferir no consumo metabólico de oxigênio, no catabolismo proteico e no equilíbrio hidroeletrolítico, além de atrasar a cicatrização e imunossuprimir o paciente, levando à deterioração dos sistemas cardiovascular, respiratório e gastrointestinal (Wetmore e Glowaski, 2000).

Apesar da pouca quantidade de estudos que relatam a dor pós-operatória associada ao procedimento de mastectomia em cadelas (Steagall et al., 2006; Sarrau et al., 2007; Nakagawa et al., 2007; Gakiya et al., 2011), é válido ressaltar que uma terapia multimodal deve ser estabelecida para o tratamento adequado da dor nesses pacientes.

A anestesia infiltrativa por tumescência é uma técnica muito empregada na medicina humana e tem se expandido a diversos procedimentos cirúrgicos em virtude da sua efetividade e relativa segurança no tratamento antiálgico (El Khatib, 2011). Atualmente, a tumescência vem sendo empregada na medicina veterinária como técnica adjuvante da anestesia geral em procedimentos de mastectomias em cadelas, com a finalidade de melhorar o controle da dor durante o procedimento anestésico (Lopes e Almeida, 2008).

Assim, com este trabalho, procurou-se aquilatar, comparativamente, a resposta álgica pósoperatória em cadelas submetidas à mastectomia unilateral, utilizando-se protocolo convencional de anestesia ou a associação desta à técnica de tumescência, de modo a se estabelecerem valores confiáveis para a espécie em teste e determinarse a viabilidade ou não da utilização de um ou outro método anestésico.

\section{MATERIAL E MÉTODOS}

Este estudo foi aprovado pela Comissão de Ética e Bem-Estar Animal (CEBEA) sob protocolo $n^{\circ}$ 021320 .

Foram utilizadas 20 cadelas (Canis familiaris), provenientes do atendimento clínico de rotina do Hospital Veterinário, de raças variadas, com idade entre sete e 12 anos (média de 9,2 anos) e peso variando entre 4 e $41 \mathrm{~kg}$ (média de $14,3 \mathrm{~kg}$ ), 
portadoras de neoplasia mamária diagnosticada previamente por meio de citologia aspirativa por agulha fina. Os animais foram aleatoriamente divididos em dois grupos de 10 cães, denominados grupo controle (GC) e grupo tumescência (GT), de acordo com a técnica utilizada em cada um deles.

Todos os animais foram submetidos à pesquisa de metástases (radiologia torácica e ultrassonografia abdominal) e aos exames de hemograma e perfis bioquímicos séricos hepáticos (fostatase alcalina - FA, alaninaaminotransferase - ALT e gamaglutaminotransferase - GGT) e renais (ureia e creatinina), além de avaliação cardíaca (eletrocardiograma e ecocardiograma).

As cadelas de ambos os grupos (GC e GT) foram submetidas à medicação pré-anestésica composta por acepromazina (Acepram ß $0,2 \%$, acepromazina, Vetnil, Louveira, SP) $(0,05 \mathrm{mg} / \mathrm{kg})$ e sulfato de morfina (Dimorf® $10 \mathrm{mg} / \mathrm{mL}$, sulfato de morfina, Cristália Produtos Químicos Farmacêuticos Ltda., São Paulo, SP) $(0,3 \mathrm{mg} / \mathrm{kg})$, administrados na mesma seringa pela via intramuscular (IM). Ainda na fase pré-operatória, foram administrados meloxicam (Meloxicam $\AA$ $15 \mathrm{mg}$, meloxicam, Eurofarma, Itapevi, SP) $(0,2 \mathrm{mg} / \mathrm{kg} \mathrm{SC})$ e cefazolina (Fazolon ${ }^{\circledR} 1000 \mathrm{mg}$, cefazolina sódica, Ariston Indústria Química e Farmacêutica Ltda., São Paulo, SP) (30mg/kg IV) como anti-inflamatório e antibiótico, respectivamente. Após o início da sedação, realizou-se tricotomia ampla de toda a região ventral do tórax e do abdômen. A indução anestésica foi feita com a administração do propofol (Propovan ${ }^{\circledR}, \quad$ propofol, Cristália Produtos Químicos Farmacêuticos Ltda., São Paulo, SP) (4mg/kg IV) e manutenção com isoflurano (Forane ${ }^{\circledR}$, isoflurano, Abbott Laboratórios do Brasil Ltda., São Paulo, SP).

Para as pacientes que compuseram o GT, foi preparada a solução composta por solução fisiológica (Fisiológico - Med Flex® Sistema Fechado, solução de $\mathrm{NaCl} 0,9 \%$, Segmenta, Ribeirão Preto, SP), mantida sob refrigeração, associada a $40 \mathrm{~mL}$ de lidocaína injetável sem vasoconstritor (Cloridrato de Lidocaína ${ }^{\circledR} 2 \%$ Sem Vasoconstritor, cloridrato de lidocaína, Hipolabor, Belo Horizonte, MG) e $0,5 \mathrm{~mL}$ de adrenalina 1:1.000 (Efrinalin $\AA$, epinefrina,
Ariston Indústria Química e Farmacêutica Ltda., São Paulo, SP). Conforme preconizado por El Khatib et al. (2011), para animais com peso abaixo de $10 \mathrm{~kg}$, utilizaram-se $250 \mathrm{~mL}$ da solução descrita, e para aqueles acima de $10 \mathrm{~kg}$, utilizaram-se $500 \mathrm{~mL}$ da mesma solução. Destas diluições, seja a $0,3 \% \quad(250 \mathrm{~mL}$ de solução fisiológica) ou a $0,15 \%$ (500mL de solução fisiológica), foram infundidos $15 \mathrm{mg} / \mathrm{kg}$ da solução tumescente com o auxílio de uma seringa de $20 \mathrm{~mL}$ e uma agulha $40 \times 12$, ao longo de ambos os lados de toda a cadeia a ser excisada, com uma média de 8 pontos de injeção.

A monitoração transoperatória da função cardiovascular, saturação de oxigênio no sangue $\left(\mathrm{SPO}_{2}\right)$, taxa de dióxido de carbono expirado $\left(\mathrm{ETCO}_{2}\right)$, frequência respiratória (f) e temperatura $\left(\mathrm{T}^{\circ} \mathrm{C}\right)$ foram realizadas por meio do aparelho Dixtal DX 2021(Dixtal® Modelo 2021 - Módulo de PA invasiva, Manaus, AM), com eletrocardiografia em derivação DII. A pressão arterial média (PAM) foi mensurada pelo método invasivo, valendo-se da cateterização da artéria auricular medial.

A técnica cirúrgica realizada em ambos os grupos foi a de mastectomia unilateral, conforme a descrição de Headlund (2008). Os procedimentos cirúrgico e anestésico foram realizados pelo mesmo cirurgião e anestesista, respectivamente.

Quando terminou o procedimento cirúrgico as pacientes foram mantidas com roupa cirúrgica, em gaiolas individuais, para que fossem monitoradas por um período de 24 horas, nos momentos que se seguem, uma, duas, três, quatro, seis, oito, 12 e 24 horas. Escores de dor utilizando-se a escala de dor da Universidade de Melbourne (UMPS) foram atribuídos em cada um desses momentos (Firth e Haldane, 1999). Os animais que atingiram escore igual ou acima de 11 pontos pela UMPS, em qualquer momento da avaliação, receberam $4 \mathrm{mg} / \mathrm{kg}$ de cloridrato de tramadol (Tramal 5\%®, cloridrato de tramadol, Hipolabor Farmacêutica Ltda., Sabará, MG) (IM) como analgesia de resgate.

A avaliação da dor pós-operatória foi realizada por um mesmo observador "cego", experiente e previamente treinado para uso da UMPS, para avaliar a dor em cadelas submetidas à mastectomia. 
Após a avaliação das primeiras 24 horas e findadas as mensurações preestabelecidas no projeto em tela, foram prescritos cloridrato de tramadol $(4 \mathrm{mg} / \mathrm{kg}$ por via oral - VO, a cada oito horas - TID), dipirona sódica $(21 \mathrm{mg} / \mathrm{kg} \mathrm{VO}$, TID) e cefalexina (30mg/kg VO, a cada 12 horas - BID) por sete dias; além de meloxican $(0,1 \mathrm{mg} / \mathrm{kg}$ VO, a cada 24 horas - SID), por três dias, e ranitidina $(2,2 \mathrm{mg} / \mathrm{kg}$ VO, BID, sete dias). Foram recomendados retorno para avaliação da cicatrização da pele e possível retirada dos pontos cirúrgicos após 10 dias.

O delineamento utilizado foi $\mathrm{o}$ de parcelas subdivididas, testando-se o fator tratamento (2 níveis) nas parcelas e o fator tempo (8 níveis) nas subparcelas, com 10 repetições. Quando havia diferença significativa entre médias, elas eram comparadas entre si pelo teste de Tukey, ao nível de significância de $5 \%(<0,05)$. Como se tratava de variáveis que não possuíam distribuição normal, estas foram modificadas, usando-se a transformação logarítmica das variáveis (log observação +1) para que as análises pudessem ser efetuadas.

\section{RESULTADOS E DISCUSSÃO}

Nas 24 horas de pós-operatório que se seguiram, ambos os grupos foram avaliados em intervalos de uma, duas, três, quatro, seis, oito, 12 e 24 horas pela escala de dor da Universidade de Tabela 3. Médias dos escores de dor das cadelas submetidas à mastectomia obtidos por meio do tempo para os dois grupos

\begin{tabular}{cccccccccc}
\hline \multirow{2}{*}{ Grupos } & \multicolumn{7}{c}{ Tempo (em horas) } \\
\cline { 2 - 9 } & 1 & 2 & 3 & 4 & 6 & 8 & 12 & 24 & Média \\
\hline $\begin{array}{c}\text { Grupo } \\
\text { convencional } \\
\text { Grupo }\end{array}$ & $6,000 \mathrm{aA}$ & $5,600 \mathrm{aA}$ & $5,800 \mathrm{aA}$ & $5,700 \mathrm{aA}$ & $4,800 \mathrm{aA}$ & $5,800 \mathrm{aA}$ & $5,700 \mathrm{aA}$ & $5,000 \mathrm{aA}$ & $5,550 \mathrm{~A}$ \\
$\begin{array}{c}\text { tumescência } \\
\text { Média }\end{array}$ & $3,700 \mathrm{aA}$ & $5,000 \mathrm{abA}$ & $6,400 \mathrm{bA}$ & $5,900 \mathrm{abA}$ & $6,300 \mathrm{bA}$ & $5,500 \mathrm{abA}$ & $6,600 \mathrm{bA}$ & $6,700 \mathrm{bA}$ & $5,763 \mathrm{~A}$ \\
\hline
\end{tabular}

As neoplasias mamárias em cadelas apresentam grande incidência na prática veterinária de pequenos animais e se caracterizam por alta morbidade e mortalidade (Stratmann et al., 2008). A excisão cirúrgica é o tratamento de escolha nestes casos (White, 2007). Como a dor decorrente desse procedimento é intensa, merece a preocupação do médico veterinário (Steagall et al., 2006; Sarrau et al., 2007).
Melbourne. Os resultados obtidos estão compilados nas Tab. 1, 2 e 3.

Tabela 1. Escores de dor das cadelas submetidas à mastectomia unilateral. Grupo convencional

\begin{tabular}{ccccccccc}
\hline \multirow{2}{*}{ Indivíduo } & \multicolumn{7}{c}{ Tempo (em horas) } \\
\cline { 2 - 9 } & 1 & 2 & 3 & 4 & 6 & 8 & 12 & 24 \\
\hline 1 & 9 & 6 & 5 & 6 & 6 & 4 & 4 & 4 \\
2 & 11 & 9 & 9 & 9 & 7 & 10 & 8 & 6 \\
3 & 7 & 8 & 8 & 6 & 6 & 9 & 7 & 8 \\
4 & 4 & 3 & 5 & 6 & 4 & 6 & 4 & 4 \\
5 & 7 & 6 & 7 & 7 & 3 & 3 & 5 & 3 \\
6 & 3 & 3 & 3 & 3 & 3 & 4 & 4 & 3 \\
7 & 2 & 4 & 7 & 7 & 6 & 8 & 9 & 4 \\
8 & 7 & 7 & 5 & 5 & 5 & 4 & 4 & 5 \\
9 & 5 & 5 & 3 & 3 & 3 & 5 & 5 & 5 \\
10 & 5 & 5 & 6 & 5 & 5 & 5 & 7 & 8 \\
\hline
\end{tabular}

Tabela 2. Escores de dor das cadelas submetidas à mastectomia unilateral. Grupo tumescência

\begin{tabular}{ccccccccc}
\hline \multirow{2}{*}{ Indivíduo } & \multicolumn{7}{c}{ Tempo (em horas) } \\
\cline { 2 - 9 } & 1 & 2 & 3 & 4 & 6 & 8 & 12 & 24 \\
\hline 1 & 6 & 10 & 10 & 10 & 9 & 4 & 8 & 9 \\
2 & 0 & 2 & 5 & 4 & 4 & 6 & 6 & 3 \\
3 & 5 & 3 & 5 & 6 & 8 & 5 & 5 & 6 \\
4 & 5 & 5 & 6 & 5 & 5 & 4 & 7 & 5 \\
5 & 5 & 7 & 5 & 3 & 7 & 6 & 7 & 5 \\
6 & 4 & 3 & 10 & 10 & 10 & 10 & 11 & 10 \\
7 & 2 & 6 & 10 & 7 & 7 & 7 & 7 & 10 \\
8 & 3 & 4 & 5 & 5 & 3 & 4 & 4 & 6 \\
9 & 4 & 3 & 3 & 3 & 4 & 4 & 4 & 6 \\
10 & 3 & 7 & 5 & 5 & 5 & 5 & 7 & 7 \\
\hline
\end{tabular}

A avaliação da dor em animais é complexa e difícil, pela ausência de entendimento da capacidade de comunicação dos pacientes ou pela própria falta de sonorização (Dohoo e Dohoo, 1996). As alterações no comportamento acabam sendo o componente principal dessa avaliação, visto que os pacientes podem apresentar alterações posturais, agressividade, entre outros sinais. Existem diversos estudos referentes aos métodos de interpretação da dor em animais, nos quais são descritas escalas de 
avaliação subjetiva que são extremamente úteis na prática veterinária (Holton et al., 2001). A utilização da escala de dor da Universidade de Melbourne (Firth e Haldane, 1999) mostrou-se satisfatória para o reconhecimento da dor neste estudo. Tal fato vai de encontro aos achados de Colantonio (2000), em que as medidas comportamentais e fisiológicas, quando tomadas em conjunto, podem ser usadas de forma confiável para avaliar o grau de dor em cães no período pós-operatório. Para Sarrau et al. (2007), saber identificar e tratar adequadamente a dor é fundamental no tratamento de animais portadores de neoplasias mamárias submetidos à mastectomia.

Após a análise das médias dos escores dos dois grupos, pôde-se observar que não houve diferença estatística $(\mathrm{p}=0,922)$. Também não houve diferença estatística ao longo do tempo para nenhum grupo nem entre os dois grupos em cada momento da coleta. Apenas dois animais apresentaram analgesia inadequada (escore 11): o animal 2 do grupo convencional apresentou, já na primeira hora de pós-operatório, aumento em $100 \%$ da frequência cardíaca basal, salivação e pupilas em midríase; e o animal 6 do grupo tumescência apresentou midríase, reação à palpação, estado mental cauteloso e vocalização intermitente às 12 horas de pós-operatório. Ambos os animais receberam o resgate analgésico com cloridrato de tramadol, na dose de $4 \mathrm{mg} / \mathrm{kg}$ (IM), para promover a analgesia efetiva.

A técnica de tumescência foi descrita em 1987, quando da infiltração de grandes volumes de uma solução diluída de lidocaína e adrenalina no tecido adiposo em pacientes humanos antes do procedimento de lipoaspiração (Behroozan e Goldber, 2005). Atualmente a solução anestésica administrada consiste em um anestésico local, um fármaco vasoconstritor, uma substância reguladora de $\mathrm{pH}$ e uma solução intravenosa estéril, que também podem estar associados a um fármaco anti-inflamatório esteroidal (Futema, 2005).

A infusão da solução tumescente foi realizada sem observação de dificuldades e pode ser realizada de maneira manual, por meio da inserção da ponta da agulha na derme em um ângulo de $30^{\circ}$, seguida pela injeção lenta da solução, conforme relatado por Futema (2005).
Tal fato exclui a necessidade de materiais especializados para a técnica, o que torna a sua prática passível de execução na rotina cirúrgica de pequenos animais. Ainda, caso haja preferência e disponibilidade, podem ser utilizadas bombas de infusão (Carlson, 2005).

Adicionalmente, foi observada uma redução visual subjetiva na intensidade de sangramento nas pacientes submetidas à técnica de tumescência, fato este previamente sugerido por Futema (2005). Não foi observada, entretanto, uma diferença entre as técnicas com relação à facilidade de retirada em bloco da cadeia mamária, conforme constatado por este mesmo autor.

Também não foram verificados sinais de neurotoxicidade, cardiotoxicidade ou necrose local às pacientes submetidas à técnica de tumescência, como foi relatado em pacientes humanos por De Jong (2000) e Humber et al. (2004). No entanto, tal observação corrobora os achados de Namias e Kaplan (1998), que não notaram tais sinais em cadelas.

Os achados observados com relação à analgesia pós-operatória rechaçam os vistos em humanos, uma vez que não se verificou diferença entre a dor em animais submetidos à anestesia geral inalatória e naqueles que receberam a técnica anestésica local de tumescência. Naquela espécie, relata-se diferença entre pacientes submetidos ou não à técnica de tumescência com relação à dor no período pós-cirúrgico (Namias e Kaplan, 1998).

Os bons escores relacionados à dor obtidos nas 24 horas de pós-operatório no presente projeto, em ambos os grupos experimentais, devem-se provavelmente à utilização da analgesia multimodal. Nakagawa et al. (2007) demonstraram que cadelas submetidas à mastectomia que receberam meloxicam, assim como no presente estudo, apresentaram uma menor pressão arterial e frequência cardíaca por até cinco dias de pós-operatório em relação aos cães do grupo que receberam solução fisiológica $0,9 \%$. Portanto, é de se esperar que cadelas que receberam o tratamento analgésico multimodal no pré-operatório apresentem menos dor pós-operatória, uma vez que a informação nociceptiva aferente é reduzida com o uso desses 
analgésicos (Lascelles et al., 1998). Isto foi observado em ambos os grupos experimentais.

Embora as vantagens da utilização da técnica de tumescência com relação à dor pós-operatória em humanos seja um fato consagrado (Namias e Kaplan, 1998), infelizmente, faltam dados sobre essa técnica em animais. Diante dos resultados encontrados, sugere-se não haver vantagens entre uma técnica ou outra, desde que seja realizada da forma correta e com suporte analgésico adequado. A aparente redução na intensidade de sangramento observada no GT poderia ser considerada uma vantagem da realização desta técnica, entretanto precisa ser melhor estudada e descrita.

\section{CONCLUSÃo}

Conclui-se que não houve diferença significativa entre a anestesia geral inalatória e a mesma anestesia associada à técnica de anestesia local por tumêscencia nas condições em que o presente estudo foi realizado. No entanto, outras vantagens, como a provável redução do sangramento transoperatório, tornam desejável a utilização da técnica de tumêscencia, ficando o seu uso a cargo da experiência e preferência do cirurgião e do anestesista. O uso da técnica de tumescência se mostrou exequível em cadelas submetidas à mastectomia unilateral. O protocolo analgésico utilizado na presente pesquisa minimizou a dor pós-operatória, de acordo com a escala de avaliação empregada, e é recomendado para cirurgias de mastectomia em cadelas.

\section{AGRADECIMENTOS}

À Fapesp, pela bolsa de pesquisa PIBIC.

\section{REFERÊNCIAS}

BEHROOZAN, D.S.; GOLDBERG, L.H. Dermal tumescent local anesthesia in cutaneous surgery. J. Am. Ac. Dermat., v.53, p.828-830, 2005.

CARLSON, O.W. Total mastectomy under local anesthesia: the tumescent technique. The Breast J., v.2, p.100-102, 2005.
COLANTONIO, D. Evaluation of postoperative pain in dogs. Disponível em: $<$ http://vip.vetsci.usyd.edu.au/contentUpload/con tent_3077/colantonio.pdf>

Acessado em: 26 Mar. 2013.

DALECK, C.R.; FRANCESCHINI, P.H., ALESSI, A.C. et al. Aspectos clínico e cirúrgico do tumor mamário canino. Cienc. Rural, v.30, p.731-735, 1998.

DAVIS, K.M.; STONE, E.A. Neoplasia da glândula mamária. In: BICHARD, S.J.; SHERDING, R.G. (Ed.). Manual Saunders Clínica de pequenos animais. 3.ed. São Paulo: Roca, 2008. p.316-320.

DE JONG, R.H. Mega-dose lidocaine dangers seen in "tumescent" liposuction. J. Clin. Monit. and Comp., v.1, p.77-79, 2000.

DOHOO, S.E.; DOHOO, I.R. Factors influencing the postoperative use of analgesics in dogs and cats by canadian veterinarians. Can. Vet. J., v.37, p.552-556, 1996.

EL KHATIB, E.M.; REPETTI, C.S.T; PIRES, P.T.A.; HATAKA, A. Técnica infiltrativa por tumescência associada à pesquisa do linfonodo sentinela na mastectomia em cadelas: estudo experimental. Rev. Nosso Clin., v.80, p.24-32, 2011.

FIRTH, A.M.; HALDANE, S.L. Development of a scale to evaluate postoperative pain in dogs. $J$. Am. Vet. Med. Assoc., v.214, p.651-659, 1999.

FUTEMA, F. Anestesia por tumescência. In: Encontro de Anestesiologia Veterinária, 7., 2005, São Luís. Anais...São Luís: [s.n.] 2005. p.88-97.

GAKIYA, H.H.; SILVA, D.A.; GOMES J. et al. Electroacupuncture versus morphine for the postoperative control pain in dogs. Acta Cirur. Bras., v.26, p.346-351, 2011.

HEADLUND, C.S. Cirurgia do sistema reprodutivo e genital. In: FOSSUM, T.W. (Ed.) Cirurgia de pequenos animais. 3.ed. Rio de Janeiro: Elsevier, 2008. p.702-774.

HOLTON, L.; REID, J.; SCOTT, E.M. et al. Development of a behavior-based scale to measure acute pain in dogs. The Vet. Rec., v.148, p.525-531, 2001. 
HUMBER, M.O.; KOCH, H.; HAAS, F.M. et al. Necrotizing fasciitis after ambulatory phleboctomy performed with use of tumescent anesthesia. J. Vasc. Surg., v.39, p.263-265, 2004.

JOHNSON, C.A. Distúrbios do sistema reprodutivo. In: NELSON, R.W.; COUTO, C.G. (Ed.) Medicina interna de pequenos animais. 4.ed. Rio de janeiro: Elsevier, 2010. p.947-949.

LASCELLES, B.D.; CRIPPS, P.J.; JONES, A.; WATERMAN-PEARSON, A.E. Efficacy and kinetic of carprofen, administered preoperatively or postoperatively, for the prevention of pain in dogs undergoing ovariohysterectomy. Vet. Surg., v.27, p.568-582, 1998.

LOPES, B.C.C.; ALMEIDA, R.M. Anestesia local no controle da dor: a técnica infiltrativa por tumescencia - revisão de literatura. Rev. Clin. Vet., v.13, p.70-74, 2008.

MORRIS, J.; DOBSON, J. Mammary gland. In: MORRIS, J.; DOBSON, J. Small animal oncology. Iowa: Blackwell Science, 2001. p.184189.

NAKAGAWA, K.; MIYAGAWA, Y.; TAKEMURA, N.; HIROSE, H. Influence of preemptive analgesia with meloxicam before resection of the unilateral mammary gland on postoperative cardiovascular parameters in dogs. J. Vet. Med. Sci., v.69, p.939-944, 2007.

NAMIAS, A.; KAPLAN, B. Tumescent anesthesia for dermatologic procedures. Am. Soc. Dermat. Surg., v.24, p.755-758, 1998.

OTONI, C.C.; RAHAL, S.; VULCANO, L.C. et al. Survey radiography and computerized tomography imaging of the thorax in female dog with mammary tumors. Acta Vet. Scand., v.52, p.20, 2010.
QUEIROGA, F.; LOPES, C. Tumores mamários caninos - novas perspectivas. Congresso de ciências veterinárias. In: PROCEEDINGS OF THE VETERINARY SCIENCES CONGRESS SPCV, 1., 2002, Oeiras. Anais... Oeiras: [s.n.] p.183-190. 2002.

SARRAU, S.; JOURDAN, J.; DUPUISSOYRIS, F.; VERWAERDE, P. Effects of postoperative ketamine infusion on pain control and feeding behavior in bitches undergoing mastectomy. J. Small Anim. Prac., v.48, p.670676, 2007.

STEAGALL, P.V.M.; TEIXEIRA NETO, F.J.; MINTO, B.W. et al. Evaluation of the isoflurane-sparing effects of lidocaine and fentanyl during surgery in dogs. J. Am. Vet. Med. Assoc., v.229, p.522-527, 2006.

STRATMANN, N.; FAILING, K.; RICHTER, A.; WEHREND, A. Mammary recurrence in bitches after regional mastectomy. Vet. Surg., v.37, p.82-86, 2008.

VAN NIMWEGEN, S.; KIRPENSTEIJN, J. Specific disorders. In: TOBIAS, K.M.; JOHNSTON, S.A. (Ed.) Veterinary surgery small animal. Missouri: Elsevier, 2012. p.13201325.

VASCELLARI, M.; BAIONI, E.; RU, G. et al. Animal tumor registry of two provinces in northern Italy: incidence of spontaneous tumors in dogs and cats. BMC Vet. Res., v.5, p.39, 2009.

WETMORE, L.A.; GLOWASKI, M.M. Epidural analgesia in veterinary critical care. Clin. Techn. Small Anim. Prac., v.15, p.177-188, 2000.

WHITE, R.A.S. Tratamento cirúrgico de distúrbios cutâneos específicos, In: SLATTER, D. (Ed.) Manual de cirurgia de pequenos animais. 3.ed. Barueri: Manole, 2007. p.339-355. 